\title{
Using Online Hotel Customer Reviews to Improve the Booking Process
}

\author{
Wojoud Al-Abdullatif \\ Al-Imam Mohammad Ibn Saud Islamic University \\ College of Computer and Information Sciences. \\ Information Systems Department, Riyadh, KSA
}

\author{
Yasser Kotb \\ Al-Imam Mohammad Ibn Saud Islamic University, \\ College of Computer and Information Sciences, \\ Information Systems Department, Riyadh, KSA. \\ *(On leave) Ain Shams University, Faculty of Science, \\ Mathematics/Computer Science Department, \\ Abbassia, Cairo, Egypt
}

\begin{abstract}
The e-commerce field has developed to the point that more and more hotel companies provide online booking services to travelers as an integral part of their business model. Increasing numbers of hotel companies now provide such services as an integral part of their business model and their guests' experiences with their hotel. Some third-party services allow customers to add comments on each hotel at the affiliated website. The current search tool features at hotel websites are based on fixed properties, allowing companies to take advantage of the huge number of available customer reviews to provide relevant information to consumers considering new services. The present research focuses on the possibility of linking customer reviews with search tools for online hotel booking and dividing the customers into categories based on their travel aims. This shall be accomplished by: 1) extracting customer reviews using opinion mining and finding hotel features that are frequently mentioned in the reviews, and 2) then analyzing those features to achieve the goal of enhancing booking processes by adding new characteristics, based on customer preferences. This research should improve online hotel booking by building a customized tool that utilizes available customer reviews at the Agoda website and matches them with users' preferences based on survey results.
\end{abstract}

Keywords opinion mining, customer reviews, hotels and features.

\section{INTRODUCTION}

There has been a significant increase in the number of consumers using the Internet to find hotel rooms and companies are always seeking better services and products to satisfy their clients or customers. People are becoming more interested in booking hotels through the Internet, preferring this method because it is much easier and faster than traditional methods. Some of the most important information available on the web can be found in the opinions expressed by users, such as through customer product reviews. Online customer reviews provide the user with quick access to information related to hotels and help them make decisions faster than ever before. More customers are now reading a variety of online reviews and using them to form opinions and make decisions. Customer reviews are a bridge between sellers and buyers. Trust in online customer reviews is growing and often, positive customer reviews increase the trust others have in a hotel. Customer reviews are also important because they often reference hotel features that do not appear in hotel descriptions. The availability of clear and precise reviews is valuable for hotel companies.

Customer reviews have not yet been studied in terms of their ability to enhance search tool results and facilitate the booking process. Currently, online hotel booking services offer database searches based on restricted features and criteria, and do not take into account the frequently mentioned aspects of customer reviews and preferences, which represent an excellent resource.

As a result, the ability to match hotel specifications with a customer's needs is limited. The descriptions and categorizations of hotels on websites do not offer enough valuable information for all customers. Sometimes, a customer wants to know more details about a hotel's characteristics. Booking processes could therefore be more helpful for customers if they provided information on a lot of features, which would also improve their search results.

Better results could be obtained by using an opinion-mining system to systematically collect and analyze information from the external business environment to aid in improving the booking process for customers.

The overall objective of the research is to facilitate and strengthen the process of hotel searches and booking for customers by extracting elements of opinions from online customer reviews of particular hotels to determine the features commented on by consumers in order to match their preferences with the best hotels.

The task is to use opinion-mining techniques [1], specifically feature-based opinion mining, to extract hotel features from customer reviews, summarize the results and use the results to achieve the objectives. This task involves many subtasks, which are described below.

1. Create groups of travelers based on the purpose of their travel (business, study, tourism and therapy) and their status (family, group or alone) with their preferred hotel features, and then use these groups as reference points during data collection. This step will be achieved by conducting a survey.

2. Perform opinion extraction by extracting opinions from web documents and gathering reviews about hotels from Agoda.com. [2] This step will be achieved by building a tool using Visual Basic .net. 
3. Perform opinion word extraction by selecting and extracting feature words from the opinions extracted in the previous step.

4. Identify the extracted hotel features as positive or negative, and then calculate the frequency with which all features extracted for each hotel are mentioned to determine the hotel features that are most important to customers, based on the survey results.

5. Link all hotels that contain the features with each appropriate group in the first step [3][4].

After finding and listing all the important hotel features based on customers' opinions, the following actions could be performed:

- Set customers' categories as a new section in website search engines.

- Allow users to select their attributes.

- Show the most important hotels based on the hotels' own categories and customer reviews.

The paper is organized into the following sections: Section 2 presents the previous work done in the area of customer opinion mining. Section 3, describes the architecture of the system we built for customer opinion mining. Section 4, contains the discussion of the findings of this study. The last section concludes the study.

\section{RELATED WORK}

Commercial use of the Internet started in the early 1990s. In that time, web servers and web browsers also saw development. That evolution led to the use of personal computers in homes and businesses for the purpose of generating network communications. These three factors facilitated the launch of e-commerce businesses. With the evolution of e-commerce, both the economy and businesses have fundamentally changed.

\subsection{Customer Reviews at E-Commerce Websites}

Significant research has been conducted in the field of online product review analysis. Chung and Tseng [5] provide reviews on discovering business intelligence from online product reviews. They discuss existing works that analyze online product reviews and critically review the existing approaches. Like as, Zhu and Zhang [6] studied the reviews' of online games and their impact on sales and found that these reviews are more influential for less popular games and games whose players have greater Internet experience. Yan et al. [7] developed a dictionary-based method to represent review textual features and used machine-learning techniques to classify the review sentiment. To address ambiguity in review text, Ding and Liu used linguistic rules to determine the semantic orientations of words in customer reviews.

\subsection{Web Mining}

Web mining is considered data mining that is accomplished by extracting useful information and knowledge from a large number of webpages on websites. There are three main types of web mining: web content mining, web structure mining and web usage mining [8].

\subsubsection{Web Content Mining}

Web content mining is the process of extracting useful information from the contents of a webpage or site. Content data corresponds to the collection of facts that a webpage was designed to convey to users. Web documents may consist of text, images, audio, video or structured records, such as lists and tables. One web content mining technique is web scraping [8].

\section{- Web Scraping}

Web scraping is defined as extracting useful information from HTML pages by parsing the webpages using specially coded programs for manipulation; it is also called data extraction. There are various levels of web scrapping, like human copy and paste, text grepping, HTTP programming, embedded web browsers, HTML parsers and web scraping software tools [9].

Nowadays, web wrappers are popular tools for extracting information from webpages. Wrappers are "specialized program routines that automatically extract data from Internet websites and convert the information into a structured format [10]." Actually, wrappers have three main functions. First, wrappers must be able to download HTML pages from a website. Second, they search for, recognize and extract specified data. Third, wrappers save this data in a suitably structured format to enable further manipulation [10].

One study proposed an XPath-wrapper induction algorithm that extracts object attribute values and does not require laborintensive training data. Research found that $40-50 \%$ of web content consists of webpage templates. Template-based sites have common properties, so pages within a website have a similar structure, and the positions of attribute values are fixed within pages and can thus be defined as paths from the root to the node called XPaths, which contain attribute values on the DOM (Document Object Model) tree of the pages [11]. To extract attribute values from pages, attribute values need to be identified at a few pages of a site. In their approach, the researchers defined a weighted XPath, which is the sum of the weights of HTML tags in the XPath, and the frequency of the XPath in webpages within sites. The inverse frequency of the XPath infers the ability of the XPath to extract the correct values from webpages it has not learned. The algorithm follows many steps: first, XPath determination, in which a list of possible XPaths is determined; second, XPath elimination, in which unimportant information is eliminated from all possible XPath candidates; third, feature calculation, in which feature values (weight, frequency, inverse frequency) are calculated for each XPath; and fourth, XPath ranking, in which XPath candidates are ranked with the features [12].

\subsubsection{Web Structure Mining}

Web structure mining is the process of discovering structured information from the web. It is based on analyzing webpages to discover useful knowledge through their hyperlinks. There are two kinds of web structure mining: hyperlink mining and document structure mining [8].

\subsubsection{Web Usage Mining}

One type of data mining is web usage mining, which is defined as "the extraction of meaningful user patterns from web server access logs using data mining techniques."

This type's aim is to discover general patterns in web access logs that have extrapolated usage patterns from the available data. It is a record of all data about user interaction with resources received from user requests. This technique of analyzing web server logs helps website administrators to understand user behavior, namely how he or she used the browser and interacted with the browser interface [9] [8]. 


\subsection{Opinion Mining}

There are several approaches to opinion mining in the current research. Opinion mining is the area of research that attempts to create automatic systems to determine human opinion, with the aim of extracting attributes and components of the object that have been commented upon in each document. It is meant to determine from text written in natural language whether the comments reflect positive, negative or neutral opinions [4]. It starts with sentiments bearing lexical items, known as "Sentiment Classification," and investigates ways to classify each review document as positive, negative or neutral. Some researchers do it at the document level, whereas others work at the sentence level. The methods are essentially based on two types of approaches: 1) corpus-based approaches that find co-occurring patterns of words to determine the sentiments of words or phrases and 2) dictionary-based approaches using synonyms and antonyms in WordNet to determine word sentiments based on a set of seed opinion words [4].

\subsubsection{Opinion Mining and Customer Reviews}

In their research, Minqing and Bing reported on their process of mining and summarizing customer reviews in three main steps. First, they mined product features that had been commented on by customers, utilizing both data mining and natural language processing techniques to perform this task. Second, Minqing and Bing identified opinion sentences in each review and decided whether each opinion sentence was positive or negative. To determine the opinion orientation of each sentence, three subtasks were performed. First, a set of adjective words was identified using a natural language processing method. Second, the semantic orientation of each opinion word was determined. Finally, the opinion orientation of each sentence was assessed [3].

\subsubsection{Opinion Mining in Hotel Customer Reviews}

In December 2012, a study applied opinion mining to data from travel review sites and examined how the results of sentiment analysis of textual reviews can be visualized using Google Maps. The researchers used SentiWordNet in their application then found and extracted six features from Booking.com and Trip Advisor: "breakfast," "staff," "service," "clean," "location" and "Internet." This led to the provision of "good" and "bad" hotels as geographical areas, based on hotel reviews [13].

In 2011, system architecture for the opinion-mining framework was proposed, which consisted of two main modules: language resource construction and opinion mining modules. In their experiment, Kasper and Vela, collected hotel reviews from the Agoda website to generate a feature-based summary and comparison between hotels, based on customer reviews in Thailand. The system, called HotelOpinion, asks users to identify the city where they wish to stay. The system then displays all of the hotels in that specific city. Users can then select the name of the hotel where they prefer to stay. Based upon the user's selection, the system classifies and displays reviews for that particular hotel, based upon the following features determined earlier: "service," "hotel condition," "location," "food," "room condition," "facilities" and "price" [14].

Kasper and Vela presented a web-based opinion-mining system for hotel reviews. This system is capable of detecting and retrieving reviews on the web. The target users for this system are hoteliers who want to obtain actual overviews and summaries of textual comments about their hotel(s) on the web. With the acquisition system, data can be retrieved from the web and then stored in the hotel database and transferred to the analysis system, which is handled by a web crawler. A content extraction module is applied to the retrieved target page to extract the relevant textual content of the review and other information about the reviewer.

The analysis system checks the language to filter out reviews in other languages (this system is only available in German) and it is stored in the review database. Then the review text is segmented into sentences. Next the statistical polarity classifier divides the segments into subjects. The IE system is designed to find results regarding the following matters: the topic of the review segment, the dimension of evaluation, the dimension value and the polarity of the evaluation. After that, moving to finer-grained analysis of the polarity and the topic of the review, the linguistic information extraction is performed. Finally, the results and segments are stored in the results database [15].

\section{METHODOLOGIES}

This section presents the different methodologies utilized in this research. This research is mainly descriptive and applied research, wherein customer reviews of online hotel are evaluated for their effectiveness when used to improve searching tools. This has a quantitative research component focused on gathering hotel features and measuring the frequency of each feature mentioned in customer reviews of online hotels.

The main purpose of this work is to enhance customer service in hotel booking systems by finding the appropriate and best hotels for all travelers based on two factors: traveler type (category) and customer reviews. The target is to find the most important hotel features from the customer's point of view and match them with customer attributes.

This research proposes an opinion-mining tool that provides users a quality feature-based summary, based on opinions about hotels from ordinary customers on the web. Therefore, the tool must achieve two important goals: (1) gather opinions from different hotels on the Agoda website and (2) prepare a feature-based summary from the opinions retrieved in (1), which consists of: hotel name, feature name and the number of opinions analyzed. Then, the system must classify the sentiment associated with each of them (either negative or positive), along with text fragments where these classified features were mentioned, as in Figure 1.

\subsection{Travelers and Hotel Attributes}

There are many types of travelers in the world based on their purpose for traveling and each type prefers specific hotel features. Dividing travelers into categories leads to accurate and satisfying results. The type of traveler an individual is depends on the purpose of travel (business, study, tourism or therapy) and the status of the traveler (family, group or alone, as a couple for a honeymoon or any other).

Travelers have changed regarding which hotel attributes they care about and nowadays, there are many features available at hotels. Based on a multitude of studies, 173 attributes were extracted, with very similar items classified together. In general, after classification and summarization, there are 70 popular hotel features.

A survey including these features was conducted to determine customers' preferences. The survey actually contained two questions; first, consumers were asked to identify their type of travel (category), as mentioned in the previous section in the 
customer data subsection. Then, as part of the second question, all possible hotel features were listed and respondents were instructed as follows: "Based on your category, choose your preferred features." The questionnaire was distributed and more than 100 responses were gathered. The results of the questionnaire linked the travelers' categories with their preferred hotel features. Each type of traveler was grouped with hotel features that were saved for later use.

\subsection{Opinion Mining Tool}

To achieve the goal of finding the best hotel features for customers based on others' opinions, the process required building a tool that accessed the necessary pages and obtained responses that contained the required information. In fact, hotel information needs to be extracted with customer reviews from hotel pages, such as the country, city, hotel name, number of reviews, reviews, etc. This tool extracted reviews from the Agoda website.
The first step in the extraction process was to filter the desired links from the Agoda website for a specific structure to access the webpages, so undesirable strings had to be deleted from the links. The next step was to find out how the Agoda website stores information about hotels at each page and determine the exact attribute name (hotel name, hotel address, reviews, customer name) used at the hotel pages.

As in the pseudo code appearing in figure 2, the goal was to obtain a webpage from the Agoda website. To do so, an http web request was sent to the URL of the Agoda website through passing hotel IDs that transferred the user to the Agoda website page. Next, the html DOM selector was utilized to load the document on the page and then perform a select node. All the necessary hotel attribute values in the document were collected using string parsing and the condition that all hotels must have more than 500 reviews. The append format was also used to append the string returned by processing a composite format string. The results were stored in the HOTEL database for further use.

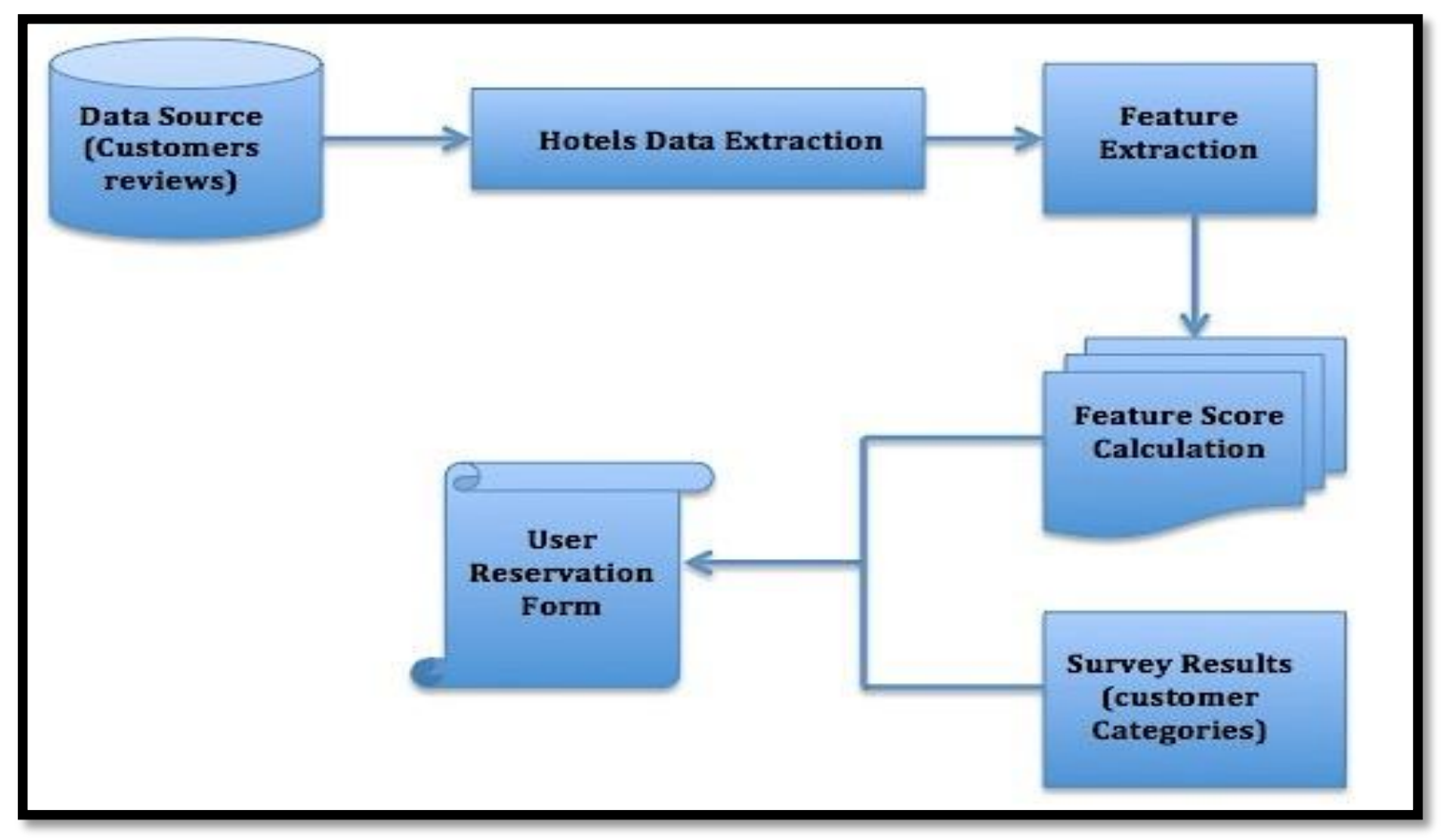

Figure 1: Overall methodologies 


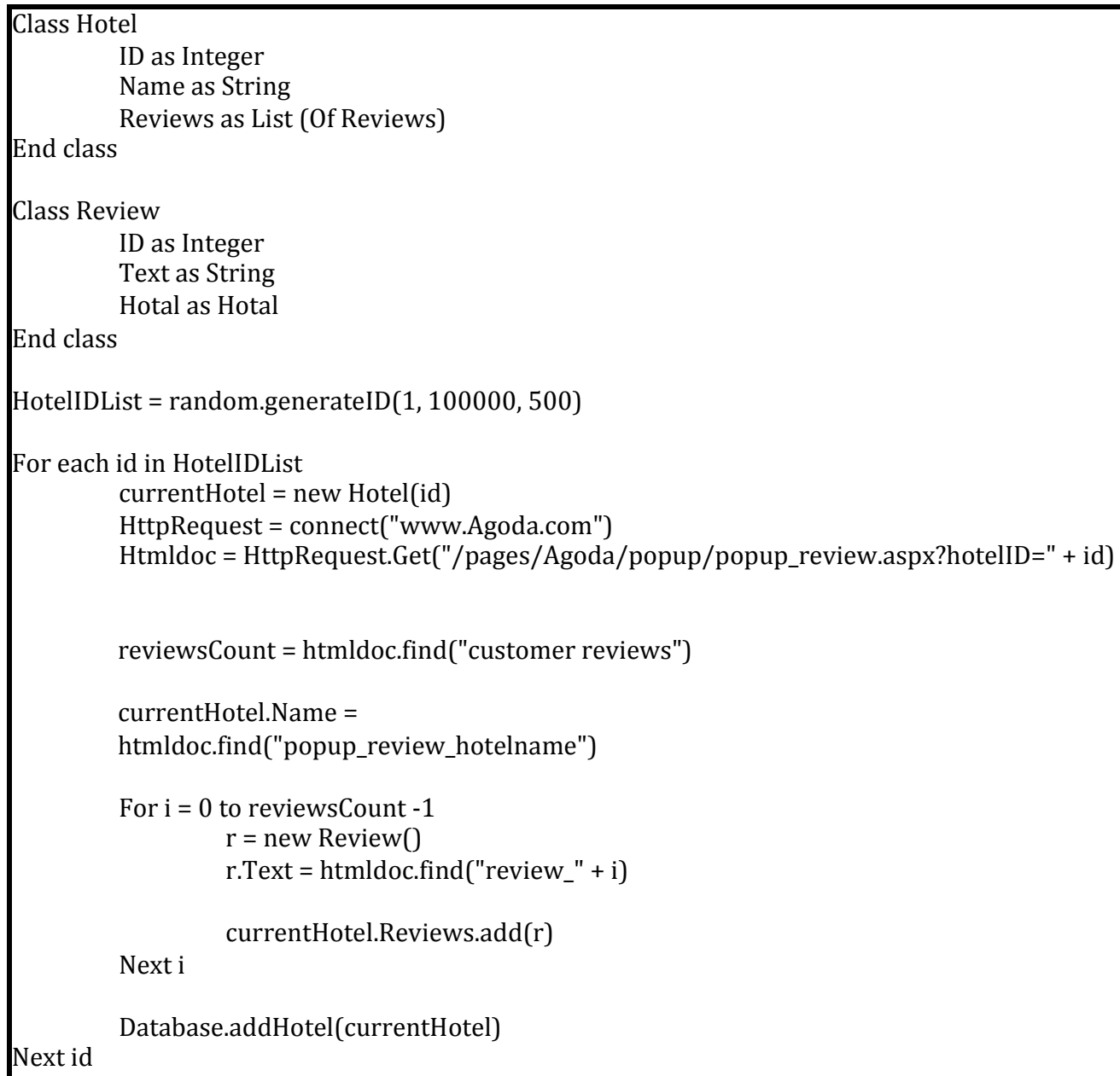

Figure 1: Pseudo code of extraction process

The process of designing an opinion-mining tool for this research was divided into four steps. The first step was to analyze the HTML page, the second step was to extract customer reviews, the third step was to analyze customer reviews and also calculate the score for hotels, and the fourth step involved the user interface, which retrieves the user query and presents the best results.

\subsubsection{Score calculation}

The aim of score calculation is to find the best hotel based on the frequency of specific features. The number of occurrences of an event in the given domain is called the frequency of that event. Since there were both positive and negative reviews, the polarity of each hotel feature cited by customers was identified as either positive or negative [3].

A negative comment on a hotel feature not mean that the user does not like anything about the hotel and a positive comment does not mean that the user liked everything about the hotel. The word "not" was used to signal negative opinions, meaning that if the feature was prefixed with the word "not," then it was considered a negative feature.

Based on that, each feature received a score; the positive feature was $(+1)$ and the negative feature was $(-1)$, so the calculation technique for hotels is the sum of the features score [16].
The equations for this calculation are described below.

First, the score value of hotel features from all customer reviews of each hotel had to be counted. This calculation was circled for each feature of each hotel.

For each feature of each hotel:

$$
\text { Feature }_{\text {Count }}=\Sigma \text { of hotel feature scores }
$$

Then, the maximum feature value among all hotels are found:

$$
\text { Feature }_{\text {Max }}=\operatorname{MAX}\left(\text { Feature }_{\text {Count }}\right)
$$

Then, the equation to find the best hotel, based on equations (1) and (2), is:

$$
\text { Hotel }_{\text {Score }}=\frac{\text { Feature }_{\text {Count }}}{\text { Feature }_{\text {Max }}} \times 100
$$

\section{EXPERIMENTAL RESULTS}

\subsection{Extract Hotels and Customer Reviews}

After running the program and connecting with the Agoda website, a random search for 100,000 hotels was performed, extracting hotels that had more than 500 reviews. A total of 1,290 hotels matching this condition were obtained from the 100,000-hotel sample. 
In the second step, reviews of previously found hotels were extracted and then stored in the Customer Review table. A total of 646,000 reviews from 1,290 hotels was actually extracted, including comments, traveler type, date of stay, member score review title and member name.

\subsection{Analyzing customer reviews}

After retrieving opinions and storing them in the database at the Customer Review table, every feature from the Customer Review table was extracted by using the Tag table and storing them in the Customer Review Tag table. So, every feature is linked with customer reviews. At the same time, the polarity of the features was reviewed and valued as either positive $(+1)$ or negative $(-1)$.

\subsection{User Interface}

This research produced a web-based user interface that enables users to find the best hotel for them in a simple way.

A simple website called "Hotel Ranker" was designed for this purpose. The hotel ranker website uses a very simple method to present information to users and allow them to book the best hotel. It contains two main pages: one for users to find based on the hotel score calculation. The result table contains the country, the city the hotel is located in and hotel names with their rating percentages; the hotel with the highest percentage is the best one. The user can also click on the hotel name to go to the hotel page.

At the administrator page, the administrator can manage categories, features and hotels in different ways.

\subsection{Results}

The survey results show that people in our society differ in their preferences regarding hotel features, based on the aim of their trip. In addition, travelers are divided into categories based on the hotel features sought. Therefore, that information was extracted from the survey responses and then stored in the hotel database for use with the hotel-ranking tool.

This research improves the online hotel booking process by providing a customized tool that uses available customer reviews from the Agoda website and matches them with users' preferences, based on the survey results.

This facilitates the booking process because searching for specific hotels is more precise than sorting through a huge

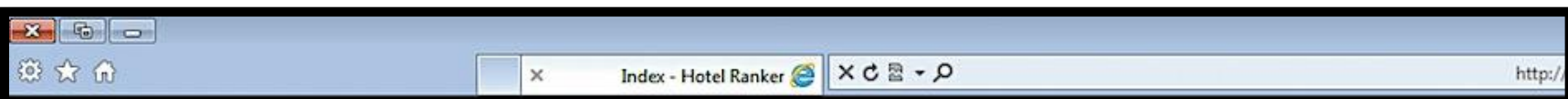

Hotel Ranker

\section{Hotels Match}

Here you can define your preferances, and search the hotels that best suits you!

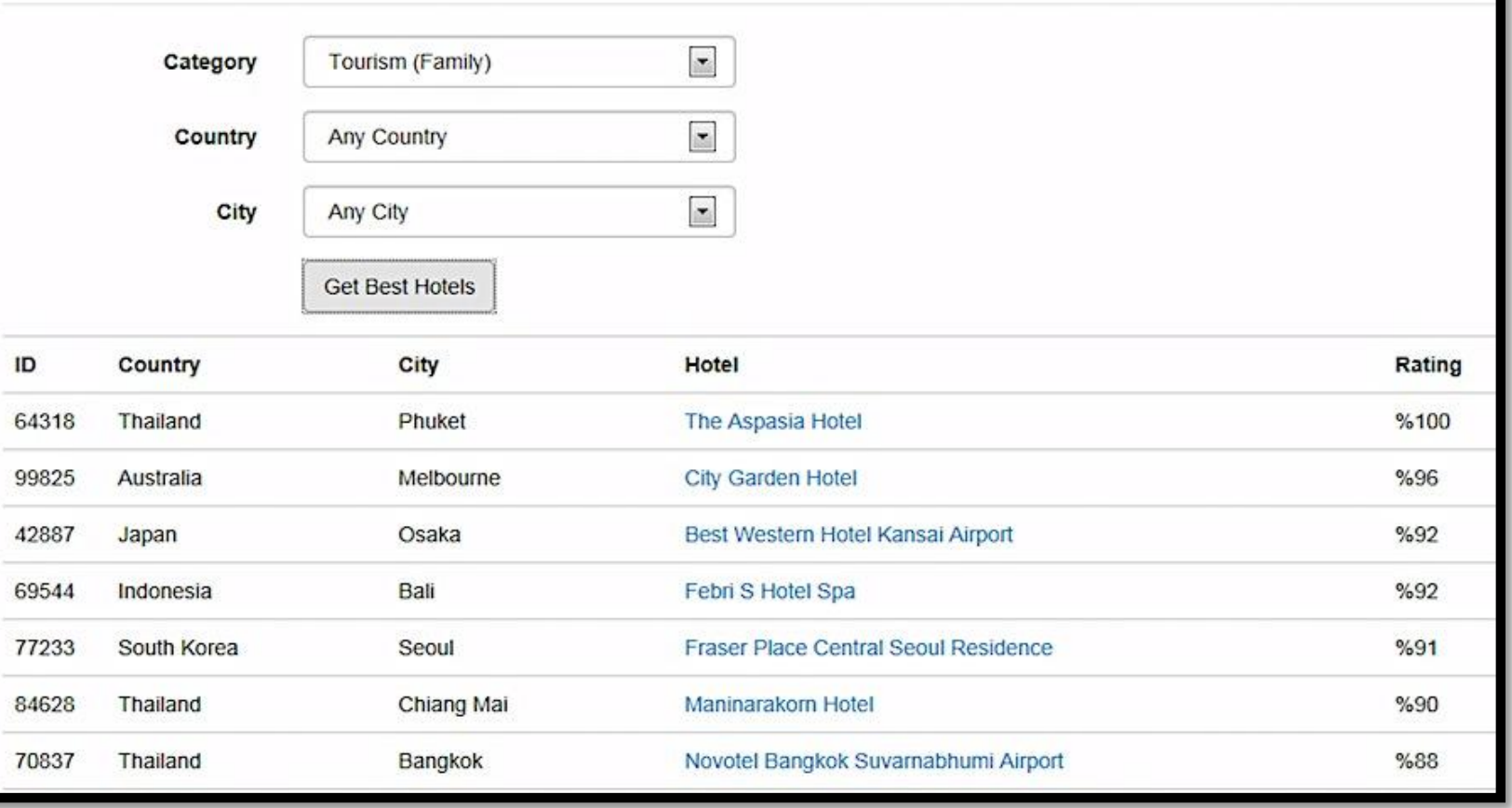

Figure 3: Searching for hotel results

the hotels and the other for the website administrator who controls and manages the whole website.

As shown in Figure 3, at the user page, the user has to select the category, the country and the city to find the best hotel, and then hotels will appear for them in descending order number of hotels.

To evaluate the effectiveness of the algorithm that determine the polarity of opinions, the program divided the reviews as positive and negative to provide accurate results for customers 
as in figure 4 , if the value is (1) this mean the review was positive and if the value is (-1) this mean the review was negative, as in figure 4.

Additionally, the program uses the frequency of words to determine the most important hotel feature from the customer's point of view is the feature "clean".

To evaluate the effectiveness of hotel extraction algorithm, the hotels score for each category are analyzed as presented in figure 5 it shows the case of customer travel as student with family (category), to Singapore.

The search results at the ranker website utilizing customer reviews are more efficient than regular search methods. Dividing customers into categories and using their opinions to provide them the best hotels leads to an enhanced booking procedure.

The ranker website provides hotels in a ranked manner so the user can more easily find the best results. Users can also go to the hotel page directly from the results table.

\section{CONCLUSION}

Opinion mining has become an interesting research area due to the availability of a huge amount of user-generated content, such as booking websites, forums and blogs. In this paper, the focus was on customer reviews available at the Agoda website; a tool that extracted those opinions was created, and the opinions were stored in the database and then analyzed. Customers were also surveyed about their preferred hotel features in the questionnaire.

This vital information was compiled to build the hotel ranker tool. In addition, the ranker website utilizing the extracted information was created, allowing the user to search for hotels based on category and receive optimal results.

The major goal of marketing is to understand the consumer and to influence buying behavior, which is achieved by providing the final piece of work: the web-based user interface that enables users to select their preferred category, country and destination city, and then find the hotel that best matches their preferences.

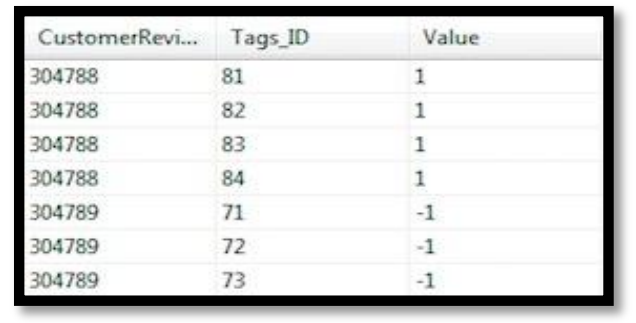

Figure 4: Negative and positive reviews

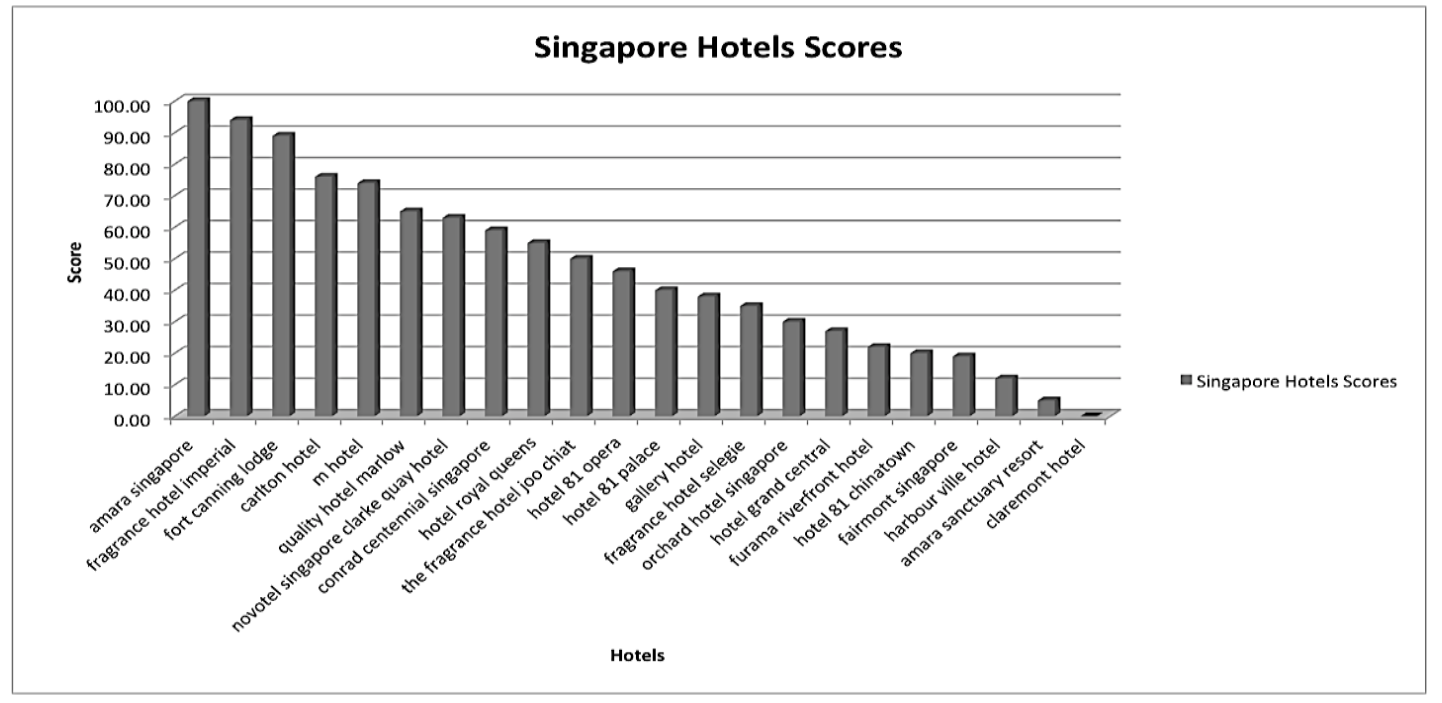

Figure 5: Effectiveness of hotel extraction and calculation

\section{REFERENCE}

[1] A. Buche, M. B. Chandak, and A. Zadgaonkar, "Opinion Mining and Analysis: A Survey," Int. J. Nat. Lang. Comput., vol. 2, no. 3, pp. 39-48, 2013.

[2] “AGODA.” [Online]. Available: http://www.agoda.com.

[3] M. Hu, B. Liu, and S. M. Street, "Mining and Summarizing Customer Reviews," in ACM, 2004, pp. 168-177.
[4] T. Bhuiyan, "QUT Digital Repository: State-of-the-Art Review on Opinion Mining from Online Customers , Feedback," in Proceedings of the 9th Asia-Pacific Complex Systems Conference 09, 2009, no. November, pp. 4-7.

[5] W. Chung and T.-L. (Bill) Tseng, "Discovering business intelligence from online product reviews: A ruleinduction framework," Expert Syst. Appl., vol. 39, no. 15, pp. 11870-11879, Nov. 2012. 
[6] X. (Michael) Z. Feng Zhu, "Impact of Online Consumer Reviews on Sales: The Moderating Role of Product and Consumer Characteristics," J. Mark., vol. 74, no. 2, pp. 133-148, 2010.

[7] H. C. Yan Dang, Yulei Zhang, "A Lexicon-Enhanced Method for Sentiment Classification: An Experiment on Online Product Reviews," IEEE Intell. Syst., vol. 25, no. 4, pp. 46-53.

[8] J. Srivastava, P. Desikan, and V. Kumar, "Web Mining Concepts , Directions," In Foundations and Advances in Data Mining Studies in Fuzziness and Soft Computing, vol. 180, 2005, 2005, pp. 51-71.

[9] S. K. Malik and S. Rizvi, "Information Extraction Using Web Usage Mining, Web Scrapping and Semantic Annotation," in 2011 International Conference on Computational Intelligence and Communication Networks, 2011, pp. 465-469.

[10] S. Kuhlins and R. Tredwell, "Toolkits for generating wrappers," in the International Conference NetObjectDays on Objects, Components, Architectures, Services, and Applications for a Networked World, 2003, pp. 184-198.
[11] "Document Object Model (DOM) - Web API Interfaces | MDN," Mozilla Developer Network and individual contributors, 2014. [Online]. Available: https://developer.mozilla.org.

[12] N.-K. Tran, K.-C. Pham, and Q.-T. Ha, "XPath-Wrapper Induction for Data Extraction," 2010 Int. Conf. Asian Lang. Process., pp. 150-153, Dec. 2010.

[13] E. Bjørkelund and T. H. Burnett, "A Study of Opinion Mining and Visualization of Hotel Reviews," in ACM, 2012, pp. 229-238.

[14] A. Kongthon, C. Haruechaiyasak, C. Sangkeettrakarn, P. Palingoon, and W. Wunnasri, "HotelOpinion: An Opinion Mining System on Hotel Reviews in Thailand," in Technology Management in the Energy Smart World (PICMET), 2011 Proceedings of PICMET '11, 2011, pp. $1-6$.

[15] W. Kasper and M. Vela, "Sentiment Analysis for Hotel Reviews," in Linguistics-Applications Conference, 2011, vol. 231527, pp. 45-52.

[16] F. Mattosinho, "Mining Product Opinions and Reviews on the Web," Technische Universitat Dresden, 2010. 